\section{'Peacock' viscous fingers}

SIR - Viscous fingering is a growth phenomenon resulting in intricate patterns that arise from the SaffmanTaylor instability ${ }^{1}$, manifested when a low-viscosity fluid displaces another with a higher viscosity. Although the phenomenon has been known for many years, the ability of these fingers to develop fractal patterns has only recently been realized ${ }^{2}$. Recently, at the Hotel Los Lebreros in Sevilla, Spain, I came across an example of viscous fingering over much greater length and timescales than I had ever seen before.

According to the waiters, the history of the 'experiment' is as follows. Several years ago the hotel coffee room was closed off with a large anticrack window divided into several $5 \times 5 \mathrm{~m}$ panes. Each pane was made up of three parallel glass plates separated from each other by a very thin film of polyvinyl. The upper and lower edges of the three glass sets were sealed to a frame by a layer of silicone (Fig. 1), while the edges were sealed by the same material to adjacent anticrack window panes. But several small holes in the paraffin covering the window edges allowed air to penetrate the base, gaining access to the polyvinyl between the glasses. Thus, an unplanned but potential Hele Shaw cell ${ }^{3}$ was created, the result being an example of what we would call "dynamic fractal self-decoration": after a few months, some fingers appeared in the window. The fingers later split, creating a branched structure and today, 11 years later, three beautiful fractal fans up to $35 \mathrm{~cm}$ in radius, which the waiters call "the peacocks", decorate the coffee room of the hotel (see Fig. 2).

This example of viscous fingering is interesting for reasons other than curiosity and beauty. First, there is no doubt that the process is triggered by temperature. This is supported by the fact that

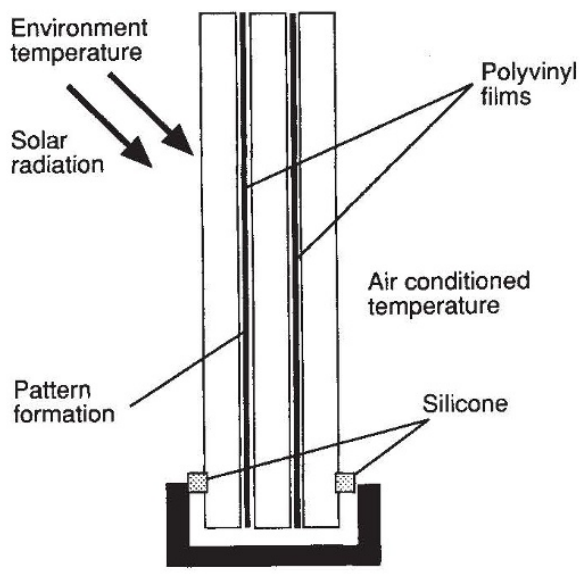

FIG. 1 Cross section of the anticrack window. the pattern is restricted to the outer film, that is, the one that takes the brunt of the climate and is unprotected by the air conditioning inside the hotel. In addition, those window panes not facing the street are unaffected. The explanation can be provided by considering that the affected window panes are oriented toward the south and sunshine in Sevilla can cause the temperature of the outer film to rise above $60^{\circ} \mathrm{C}$. This temperature must be higher than the threshold temperature at which the polyvinyl film becomes a viscous fluid. Under these conditions, the window becomes a perfect Hele Shaw cell, in which two mechanisms can account for the generation of these fractal patterns. One is the invasion of air through an initial hole pushing the viscous thin film and the other is the progressive separation of the glass plates due to thermal constraints, which is known to produce a dielectric breakdown pattern ${ }^{4}$. The perfect semicircular envelope of the fractal pattern can be explained by the interaction of both mechanisms. To my knowledge, this is the first evidence of a thermally activated Saffman-Taylor instability.

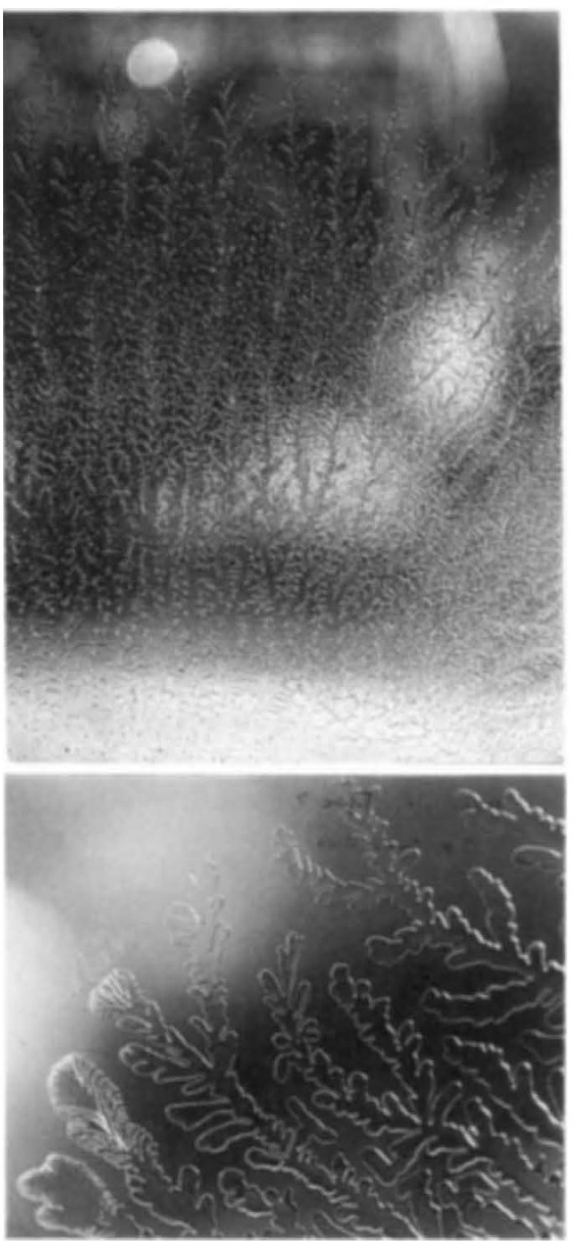

FIG. 2 Top, view of the 'peacock' pattern; bottom, detail of the fingering.
Second, the 'peacock' patterns at Los Lebreros represent an example of a large-scale viscous-fingering experiment. One can calculate the mean velocity of the tip propagation to be about $0.1 \mathrm{~mm}$ per day. So, the viscous film should be immiscible with air, because the characteristic length of the fluid displacement is similar to the characteristic diffusion length, although no diffusion patterns are observed. Even more surprising is the fact that this Hele Shaw cell should work discontinuously because the thermally driven viscoplastic behaviour of a polyvinyl film is a reversible phenomenon. Accordingly, during the night, when the temperature does not exceed $30{ }^{\circ} \mathrm{C}$, the process is dormant. The same can also be expected for longer periods during the winter. This intermittency could be the source of the banding structure observed.

Instituto Andaluz de Geología

Mediterránea,

CSIC Universidad de Granada,

Granada 18002, Spain

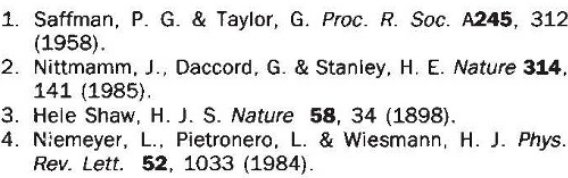
Rev. Lett. 52, 1033 (1984)

\section{CFTR mechanism}

SIR - In his recent News and Views article $^{1}$, Wine discussed evidence ${ }^{2,3}$ that expression of the cystic fibrosis transmembrane conductance regulator (CFTR) carrying the common $\Delta$ F508 mutation in non-epithelial cells results in appearance at the cell membrane of protein with chloride-channel activity. The new data showed that channel activ ity could be stimulated by a mixture of cpt-cyclic AMP, forskolin and the methylxanthine IBMX.

We had previously reported $^{4}$ that IBMX can stimulate CFTR function. We have since found that the action of IBMX is unlikely to be mediated by effects on cellular cyclic AMP levels ${ }^{5}$. The actions of methylxanthines, in particular $\mathrm{Ca}^{2+}$ mobilization or adenosine receptor antagonism, should be investigated in the development of drug strategies for the treatment of cystic fibrosis.

\section{A. MCPHERSON} R. L. DORMER

Department of Medical Biochemistry, University of Wales College of Medicine,

Heath Park, Cardiff CF4 4XN, UK

1. Wine J. I. Nature 354, 503-504 (1991)

Dalemans, $W$. et al Nature 354, 526-528 (1991)

3. Drumm, M. L. et al. Science 254, 1797-1799, (1991). 4. McPherson, M. A. et al. Lancet II, 1007-1008 (1986) . McPherson, M. A. \& Dormer, R. L. Molec. Aspects Med. 12, 1-81 (1991) 